\title{
AVALIAÇÃO DA EFICIÊNCIA DE PROTÓTIPO SOLAR DE BAIXO CUSTO COM MOVIMENTO BASEADO EM SENSOR DE LUMINOSIDADE
}

\author{
I. P. M. MEDEIROS ${ }^{1}$, T. S. ANDRÉ ${ }^{2}$, S. M. VALCACER ${ }^{3}$, C. R. F. BARBOSA ${ }^{4}$ \\ Instituto Federal de Mato Grosso do Sul ${ }^{3}$, Universidade Federal do Rio Grande do Norte ${ }^{1,2,4}$ \\ isaacmedeiros.rn@gmail.com ${ }^{1}$
}

Submetido 09/02/2017 - Aceito 28/03/2020

DOI: $10.15628 /$ holos.2020.5616

\section{RESUMO}

O estudo propõe a utilização de microcontrolador para a realização do projeto de placa de sombreamento baseado na plataforma Arduino. Para tal, usa-se um sensor da luz (LDR 10mm) para proporcionar a inclinação em relação aos raios solares, de modo que o microcontrolador usado seja capaz de fazer o rastreamento do sol com o objetivo de estudar os efeitos de tal rastreamento sobre a eficiência da captação dos raios solares, gerando assim, um sinal elétrico diretamente ao responsável pelo microcontrolador. É proposto um mecanismo que tem sua orientação baseada na máxima incidência dos raios solares na superfície da célula solar, fazendo, assim, o alinhamento direcional com a placa fotovoltaica em um ângulo com azimute e altura do Sol direto com a intensidade da radiação solar. Para isso, usam-se dois servomotores e com inclinação que pode variar nos dois eixos: Norte/Sul e Leste/Oeste. Além dos servomotores utilizou-se um microcontrolador. A placa de sombreamento forma um retângulo, devido à posição dos quatro sensores de luz, para controlar componentes diretamente ligados aos servomotores. O software embarcado é feito por algoritmos que permitem ao microcontrolador determinar a correta posição do painel solar em protótipo. Inicialmente descrevem-se as características do sistema proposto e utilizando-se desse aparato, espera-se gerar energia elétrica e aumentar a potência na captação com equipamentos de baixo custo.

PALAVRAS-CHAVE: Automação, Energia Solar, Sensor LDR, Arduino.

\section{EVALUATION OF THE EFFICIENCY OF LOW-COST SOLAR PROTOTYPE WITH MOVEMENT BASED ON LUMINOSITY SENSOR}

\begin{abstract}
The study proposes the use of a microcontroller to carry out the shading board project based on the Arduino platform. For this, a light sensor (LDR $10 \mathrm{~mm}$ ) is used to provide the inclination about the sun's rays, so that the microcontroller used is capable of tracking the sun to study the effects of such tracking on the efficiency in capturing the sun's rays, thus generating an electrical signal directly to the person responsible for the microcontroller. A mechanism is proposed that has its orientation based on the maximum incidence of solar rays on the surface of the solar cell, thus making the directional alignment with the photovoltaic plate at an angle with azimuth and height of the direct sun with the
\end{abstract}

intensity of solar radiation. For this, two servomotors are used and with the inclination that can vary in the two axes: North / South and East / West. In addition to the servomotors, a microcontroller was used. The shading plate forms a rectangle, due to the position of the four light sensors, to control components directly connected to the servomotors. The embedded software is made by algorithms that allow the microcontroller to determine the correct position of the prototype solar panel. Initially, the characteristics of the proposed system are described and, using this apparatus, it is expected to generate electrical energy and increase the power in capturing with low-cost equipment.

KEYWORDS: Automation, Solar energy, LDR Sensor board, Arduino. 


\section{INTRODUÇÃO}

Atualmente, com o aumento populacional há uma crescente demanda por energia elétrica e, consequentemente, por energias renováveis. As fontes de energia renovável ganham espaço, pois, a energia proveniente dos combustíveis fósseis acabará em algum momento, além de ser uma fonte energética poluente (Medeiros, 2015).

Uma das formas mais comuns de conversão direta dessa energia em eletricidade é através de células fotovoltaicas. No entanto, uma célula estática possui limitações na captação de energia solar devido a sua imobilidade. Com isso em mente, foi pensado que, se a célula se movesse em direção à fonte de energia luminosa, ela poderia gerar mais energia. Assim os painéis solares devem ser montados em um ângulo de 10 e 10 graus mais latitude do local e se moverem em direção ao sol (Medeiros, 2015).

O objetivo da pesquisa é conseguir que o painel fotovoltaico mova-se, direcionando-se à posição de maior energia luminosa, convertendo energia térmica em elétrica. Além disso, são apresentados resultados de simulações realizadas com o uso da placa, pois a mesma faz parte de um protótipo desenvolvido para testes/simulações do uso de painéis solares.

\section{FUNDAMENTAÇÃO TEÓRICA}

\subsection{Radiação Solar no Brasil}

A maior parte do território brasileiro está localizada relativamente próxima da linha do Equador, de forma que não se observa grandes variações na duração solar do dia (EPE, 2012).

No Brasil, como esperado, a maior incidência de raios solares está na região do semiárido nordestino. Os mapas de irradiação solar para o território brasileiro foram obtidos com os usos de modelos desenvolvidos e estimados a partir de imagens de satélites meteorológicos pelo Laboratório Nacional de Energia Renovável - NREL, os valores foram medidos nas superfícies de estações distribuídas pelo território brasileiro, como percebe-se na Figura 1.

A Região Nordeste tem grande potencial para aproveitamento de energia solar (Tobergte, 2013). O mapa na Figura 1 foi elaborado a partir dos dados disponibilizados em NREL ano 2005 e publicado em ABINEE, 2012, no qual são apresentados os dados de radiação solar em kW/m²/dia.

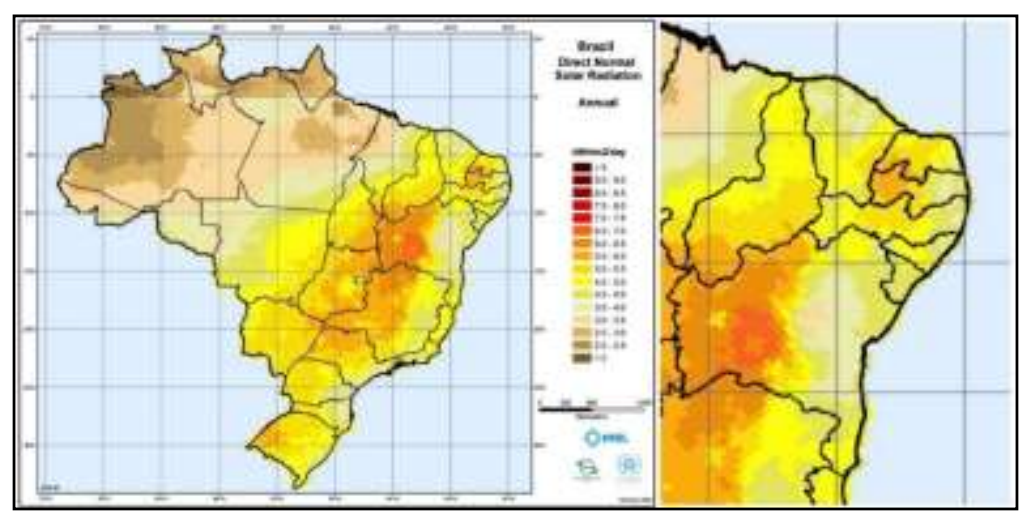


Figura 1: Irradiação total em plano cuja inclinação é igual à latitude, no Brasil (esquerda) e na Região Nordeste (direita). (NREL, 2005).

A Região Nordeste no Brasil que é representada pelas cores amarela e laranja (Figura 1) tem uma insolação média anual alta, sendo assim, viável economicamente para a geração de energia solar. A radiação solar na região nordeste varia entre 5.700 e $6.100 \mathrm{Wh} / \mathrm{m}^{2}$ dia (EPE, 2012).

\subsection{Irradiância}

A irradiância também chamada de "irradiação" é a grandeza empregada para quantificar a radiação solar em uma determinada área e pode ser expressa pela razão da potência dos raios solares pela área que de incidência desses raios (Rodrigues, 2014), Equação 1.

$P_{\text {irrad }} \frac{\text { Radiação }}{\text { Área }} \leftrightarrow \frac{\mathrm{W}}{\mathrm{m}^{2}}$

A Irradiância de $1000 \mathrm{~W} / \mathrm{m}^{2}$ é adotada pela indústria fotovoltaica como padrão para a especificação e avaliação das células fotovoltaicas (Vilalva, 2012).

\subsection{Sistema de Posicionamento}

Uma das razões que limitam o uso da energia solar é sua baixa eficiência na conversão de energia térmica em elétrica, principalmente quando as células são fixas. Segundo atuais conjunções de desenvolvimento da tecnologia, a utilização de sistemas de captação com rastreamento é uma recorrente solução para aumento da eficiência na obtenção de energia elétrica e redução nos custos.

$\mathrm{Na}$ tentativa de aumentar a eficiência na captação dos raios solares devem-se colocar os painéis solares com sua superfície sempre em posição perpendicular aos raios de Sol incidentes. Desta maneira, as mudanças na posição do Sol em relação à Terra durante o dia e no que diz respeito às estações do ano são compensadas com o reposicionamento do módulo, permitindo que mais energia seja coletada e transformada em eletricidade dentro de um determinado intervalo de tempo.

Deslumbrando o aumento da captação de raios solares faz-se uso de software que promovam a movimentação das células solares baseada na máxima incidência solar (Miyashiro, 2014).

Na maioria das aplicações de sistemas de seguimento solar, o posicionamento do conjunto de conversão de energia é baseado em sensores óticos como foto transistor, fotodiodo, LDR (Resistor Dependente da Luz) e células fotovoltaicas (Paiva, 2009).

Os sensores óticos são usados em pares e uma parede entre os dois é colocada de forma que um não veja o outro. Assim, quando estiverem igualmente iluminados pelo Sol, haverá igual incidência de radiação solar, o que garante que estarão voltados para a fonte luminosa. Quando ilumina-se uma de suas faces, o sensor aciona o motor na direção da luz até que este fique paralelo aos raios solares, significando que o conjunto está voltado para a máxima incidência solar. 


\subsection{Rastreamento}

O sistema de rastreamento irá executar o cálculo para identificar a posição do sol e para determinar os ângulos rotacionais dos dois eixos de seguimentos, a fim de conduzir o módulo fotovoltaico solar para o seguidor solar.

O LDR é um sensor de luz que usa um sinal de realimentação para assegurar que o coletor solar está seguindo o sol durante todo o tempo. São considerados parâmetros para o deslocamento: declividade terrestre, latitude, ângulo horário, dia do ano e etc. Na maioria dos aplicativos de sistema ativos de seguimento, os sensores da placa de sombreamento são suficientes para determinar a posição solares através sistema eletrônico (Alcântara, 2010).

\subsection{Posição do Sol}

A posição do sol pode ser obtida tendo em conta o ângulo da altitude e o ângulo de azimute.

O ângulo de azimute é o ângulo formado entre a direção Leste e a Oeste, da superfície horizontal, do globo terrestre. É um ângulo que vai ter uma variação entre um valor positivo e um valor negativo, para oeste é positivo e para leste negativo. A Equação 2 permite calcular o ângulo de azimute.

Azimute $=\cos _{-1}=\left|\frac{\sin \delta \cos \varphi-\cos \delta \sin \varphi \cos }{\cos \alpha}\right|$

Ao articular a altitude do Sol, é necessário referir-se ao ângulo Zenital. O ângulo Zenital é o ângulo formado entre os raios solares e a vertical (Zénite) que corresponde ao ângulo que é necessário corrigir no software para o código.

A altura do Sol é o ângulo compreendido entre o raio solar e a projeção do mesmo sobre um plano horizontal e pode ser calculada com a Equação 3.

$$
\text { Altura }_{\text {solar }}=\sin ^{-1}=[\sin \delta \sin \varphi+\cos \varphi \cos ]
$$

Na Figura 2 são apresentados os ângulos azimutal, zenital e a altura solar.
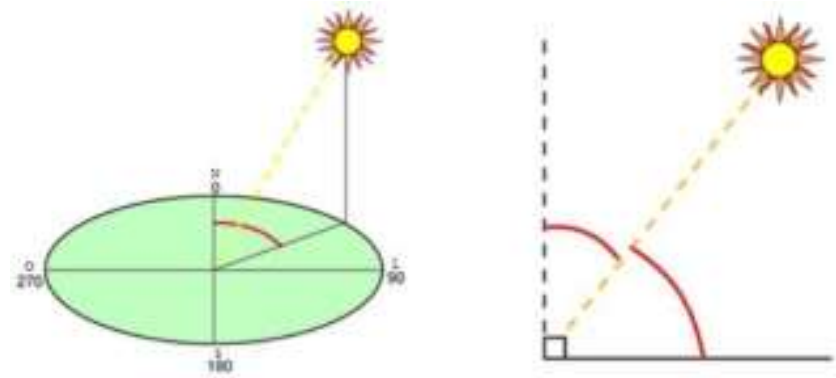

Figura 2: Rastreamentos são divididos em: altura e ângulo de azimute. (Cortez, 2013).

Ângulos causam perdas na conversão de energia de um painel fotovoltaico, o desalinhamento do mesmo com o Sol traz menor incidência do que a possível para uma 
determinada inclinação, que se traduz em perdas para o sistema. Assim surge a necessidade de um método de rastreamento solar para mover os painéis fotovoltaicos buscando sempre a maior incidência solar (Trevelin, 2014).

\section{MATERIAIS E MÉTODO}

\subsection{Metodologia}

O projeto foi dividido em duas partes: a primeira para desenvolver a parte eletrônica e a segunda para desenvolvimento do algoritmo que permita a movimentação das placas solares em virtude da máxima incidência solar. O Fluxograma 1, apresenta um resumo do projeto.

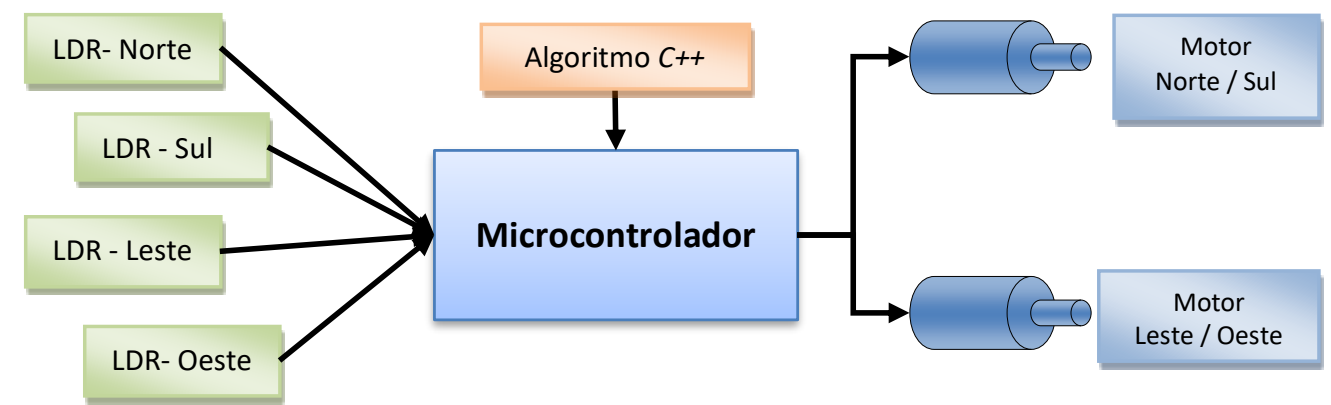

Fluxograma 1: Diagrama representativo do sistema embarcado. (Elaborada pelo autor, 2016).

Descobriu-se a existência de modelos simples, que poderiam ser adaptados aos módulos de processamento para montagem de componentes eletrônicos conectados aos sensores que fornecem informações sobre a posição do Sol. Os sinais dos sensores e o acionamento do servomotores (Moraes, 2012) são processados pelo microcontrolador utilizando um algoritmo no Arduino.

\subsection{Componentes do sistema de rastreamento solar}

Para controle do sistema de rastreamento solar utilizaram-se, basicamente, três dispositivos: sensor LDR, um microcontrolador e dois servomotores.

O funcionamento acontece da seguinte maneira: o microcontrolador recebe um sinal que vem dos sensores, interpreta e decodifica (baseado na programação) e emite uma resposta aos servomotores para movimentação e adequação à posição solar com maior incidência luminosa.

\subsection{Unidade de sensoriamento}

Sensores são dispositivos que recebem e respondem a sinais ou estímulos. Podem ser usados para medir grandezas físicas e convertem os dados obtidos em sinais elétricos. Sensores fotoelétricos são dispositivos que convertem um sinal luminoso (luz ou sombra) em um sinal elétrico ou grandeza elétrica (resistência, corrente, tensão, entre outras), que possa ser processado por um circuito eletrônico (Silva, 2014).

O controle pode ser implementado utilizando o controlador lógico programável que possibilita a programação do microcontrolador. 


\subsection{Sensor de LDR}

O sensor de LDR é um dispositivo que tem uma resistência interna variável em função do fluxo luminoso incidente em sua superfície (Silva, Cutim, Machado, 2001). Em outras palavras, o LDR é um dispositivo cuja resistência elétrica pode ser alterada devido ao fluxo incidente de radiação eletromagnética. Tem-se o máximo valor de sua resistência quando a luz é muito alta; e se a luz é baixa, a resistência no LDR aumenta.

A Figura 3 ilustra a superfície fotossensível do LDR e a Figura 4 ilustra um corte lateral do LDR. Estes apresentam uma excelente resposta fotocondutor.

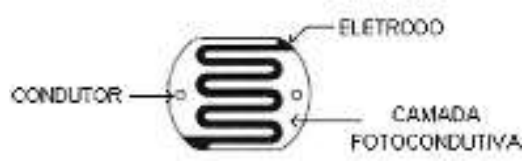

Figura 3: Superfície Fotossensível de um LDR. (Machado, 2001).

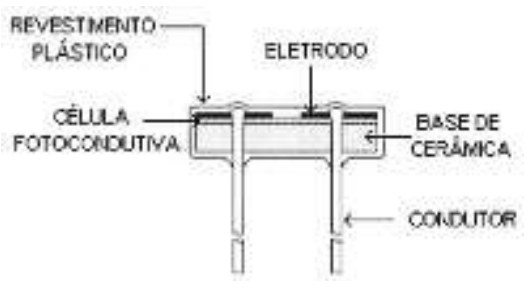

Figura 4: Corte Lateral de um LDR. (Machado, 2001).

\subsection{Estrutura de LDR}

Na pesquisa usam-se 4 sensores LDR $(10 \mathrm{~mm})$, um para cada eixo cardeal com sensores de sombreamento (Figura 5). Utilizou-se uma barreira de forma que, quando o Sol encontrar-se perpendicular à superfície dos sensores, há igual incidência de radiação solar em cada LDR e, caso contrário, o controlador irá promover a movimentação do mecanismo atuador, ou acionador, de forma que a incidência solar em cada sensor se torne igual.

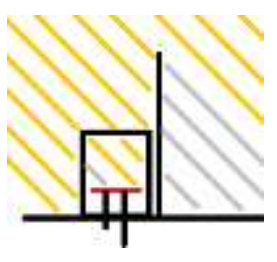

a)

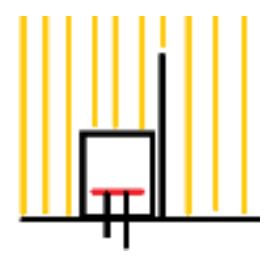

b)

Figura 5: a) A posição de incidência de o raio solar para Leste ou Oeste e b) A posição de incidência de o raio solar para céu. (Elaborada pelo autor, 2016).

A Figura 6 mostra a disposição dos sensores de luminosidade que são posicionados na base e separados por uma barreira em formato retangular.

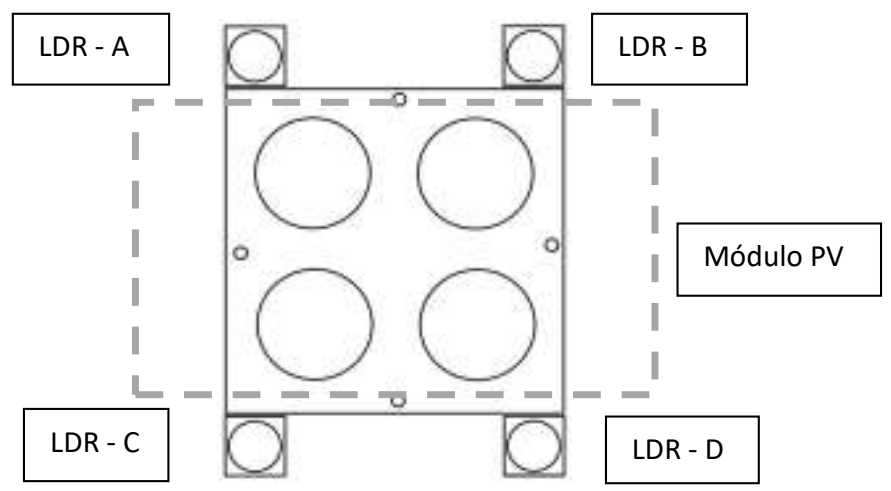

Figura 6: Vista frontal do conjunto de sensores de luminosidade. (Elaborada pelo autor, 2016). 
Os sensores LDR tem papel fundamental no protótipo. Desempenham a função de orientação solar, ou seja, são interligados com os motores, movimentando o painel fotovoltaico na direção de maior incidência solar.

A Figura 8 mostra o sensor da placa de sombreamento (formada pelo LDR mais a mangueira). Tal sensor apresenta as seguintes características construtivas: suporte em perfil tipo "T" de alumínio com dimensão de 18,5 mm e 1,58 mm de espessura; mangueira de 1/2 PVC flexível, trançada, transparente e cortada com $25 \mathrm{~mm}$ de altura, com diâmetro externo de $16 \mathrm{~mm}$. O LDR (de diâmetro de $11 \mathrm{~mm}$ ) é instalado dentro da mangueira (barreira).
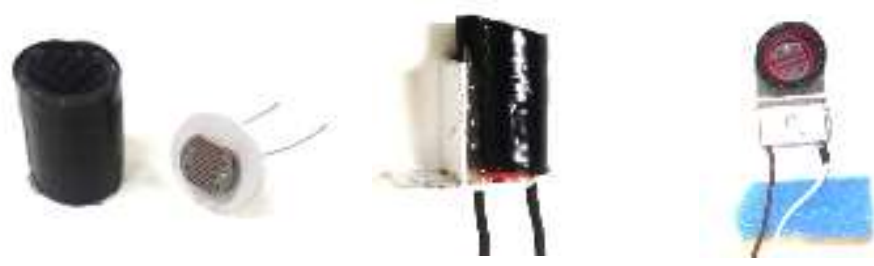

Figura 7: Placa de sombreamento e vistas. (Elaborada pelo autor, 2016).

A placa de sombreamento diminui ao máximo a reflexão da luz. Assim o ângulo de incidência dos raios solares e as saídas são as variáveis utilizadas pelo microcontrolador para a realização do controle dos movimentos em torno dos eixos vertical (azimute) e horizontal (elevação).

Para proporcionar a inclinação do dispositivo, precisou-se de uma base, com quatro placas, instaladas com acoplamento e parafuso, uma em cada lado como se pode observar na Figura 8. Ainda neste módulo, fixou-se o suporte para placa de sombreamento. O resultado final é apresentado abaixo.

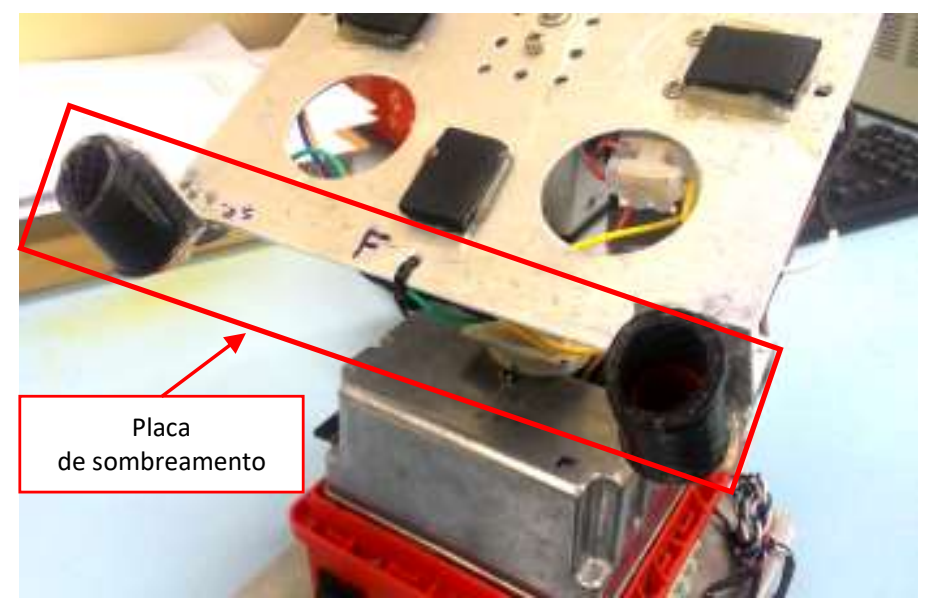

Figura 8: Suporte de palheta para uma placa de sombreamento. (Elaborada pelo autor, 2016).

\subsection{Protótipo}

O protótipo apresentado compreende apenas o sistema de rastreamento solar, sem sua aplicação em um sistema fotovoltaico. Para isso foi implementado uma estrutura que suportasse um painel fotovoltaico, com 4 sensores LDR (diâmetro de $10 \mathrm{~mm}$ cada), posicionados na estrutura em formato "retangular", um para cada borda da base onde será colocado o painel fotovoltaico. Essa mudança foi resultado de observações na literatura, uma vez que pouco se mencionava trabalhos com sensores dispostos nessa geometria (Trevelin 2014). 
Posteriormente houve a montagem dos componentes eletrônicos, eles foram colocados dentro de um módulo (caixa) de material de baixo custo para melhor acondicionamento e organização. O resultado final é apresentado na Figura 9.

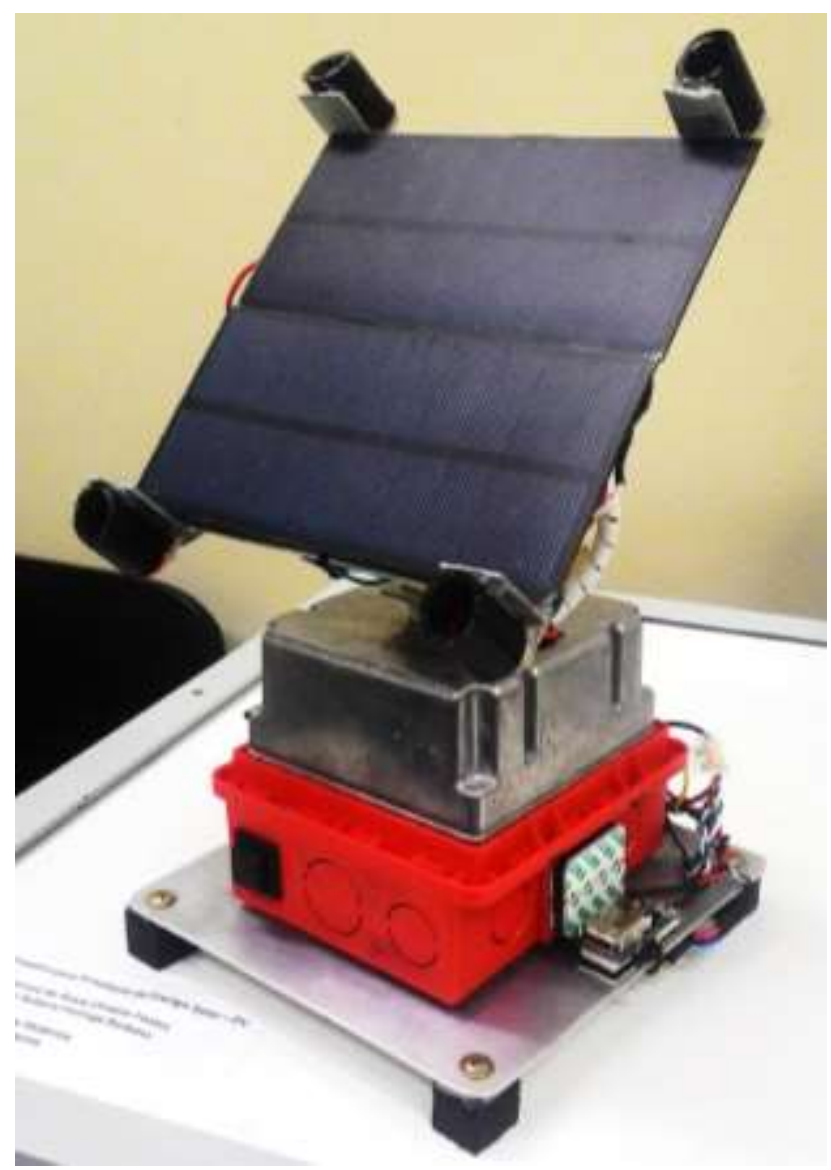

Figura 9: Vista geral, protótipo com painel fotovoltaico e instalado 4 placas de sombreamentos para sistema de rastreamento solar. (Elaborada pelo autor, 2016).

A construção da bancada apresentou um baixo custo para sua confecção. O principal componente utilizado para proporcionar sustentação ao sistema foi uma caixa de luz (interruptor) medindo, cada lado, $40 \mathrm{~mm}$. As dimensões finais são: comprimento de $220 \mathrm{~mm}$ em ca da lado e altura de $290 \mathrm{~mm}$.

\subsection{Microcontrolador e programação C++}

\subsubsection{Microcontrolador}

A plataforma encontra-se estruturada em torno de software em algoritmo; grande parte de sua operacionalização será feita em uma placa controladora, plataforma portátil e de pequenas dimensões, específica para trabalhar com sistemas móveis. A versão do Arduino ${ }^{\circledR}$ UNO R3, que também é responsável pelo controle do servomotor nas direções horizontais e verticais, trata-se de um equipamento eletromecânico para controle de rastreamento que executa um movimento de acordo com o comando específico, verificando sua atual posição pelo sol e chegando até o local desejado, diferentemente dos servomotores de rotação contínua (Medeiros, 2015). 


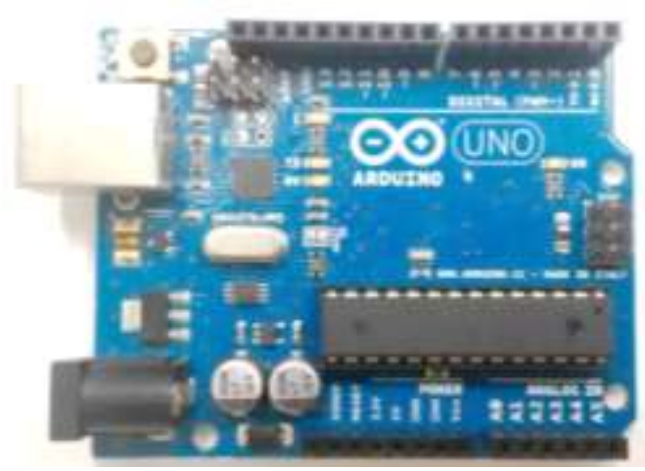

Figura 10: Módulo de microcontrolador de Arduino ${ }^{\circledR}$ UNO R3. (Arduino $\left.{ }^{\circledR}, 2016\right)$.

\subsubsection{Programação em $\mathrm{C}++$}

A linguagem de programação do microcontrolador foi feita com a utilização da linguagem de programação da plataforma de desenvolvimento do Arduino ${ }^{\circledR}$, como foi dito anteriormente, trata-se de uma linguagem em $\mathrm{C} / \mathrm{C}++$.

Para configurar o microcontrolador usa-se um código que inclui as funções desejáveis. Ao iniciar a execução do código é feita uma leitura nas portas analógicas no intervalo 0 e 1, em que está conectado o sensor que identifica uma placa de sombreamento. Nesta leitura são obtidos valores que podem variar entre 0 e 1023, de acordo com a tensão sobre os LDR. A relação entre a tensão e o valor lido na porta analógica pode ser obtida pela linguagem em C++.

As entradas selecionadas ( $A 1, A 2, A 3$ e A4) são lidas sempre que houver dois movimentos de painel. Aciona-se uma das chaves de contato, uma interrupção no movimento é realizada, rastreamento intermitente, sendo então o servomotor acionado pelo sistema.

As Figuras 11 e 12 representam os códigos de programação inicial com a declaração de variáveis.

$$
\begin{array}{ll}
\text { int sldrEI }=0 ; & / / \text { Sensor LDR Esquerdo Inferior } \\
\text { int sldrDI }=4 ; & / / \text { Sensor LDR Direito Inferior } \\
\text { int sldrES }=2 ; & / / \text { Sensor LDR Esquerdo Superior } \\
\text { int sldrDS }=1 ; & / / \text { Sensor LDR Direito Superior }
\end{array}
$$

Figura 11: Código que representa a variação dos pinos.

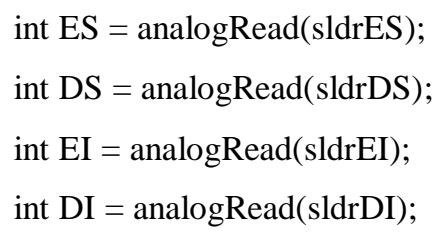

Figura 12: código de linguagem, armazenando os valores dos sensores LDR nas entradas analógicas.

O valor da incidência da luz é calculado pela diferença entre os valores lidos nas portas analógicas onde estão conectadas as duas saídas do sensor solar nos sensores (LDR). Na Figura 13 está representada a sequência de execução do código. Faz-se necessário transformar o código em um fluxograma detalhando a sequência de execução. No programa informa-se como executar os comandos de rastreamento e controle. Declara-se uma variável de controle para posições e controle. Essa variável vai contar quantas vezes o LDR se move em direção ao Sol, lado Leste para Oeste. O servomotor no início do programa assume valores preestabelecidos (configuração padrão do fabricante) e com a colocação de dados no programa, operacionaliza até reduzir o tempo a velocidade, função setup () de matemática, a trezentos milissegundos. Para movimentação dos eixos são inicializados dois blocos em vertical e horizontal, ajustados e desenvolvidos com variáveis em linguagem, com zero para início da função loop() o LDR. 


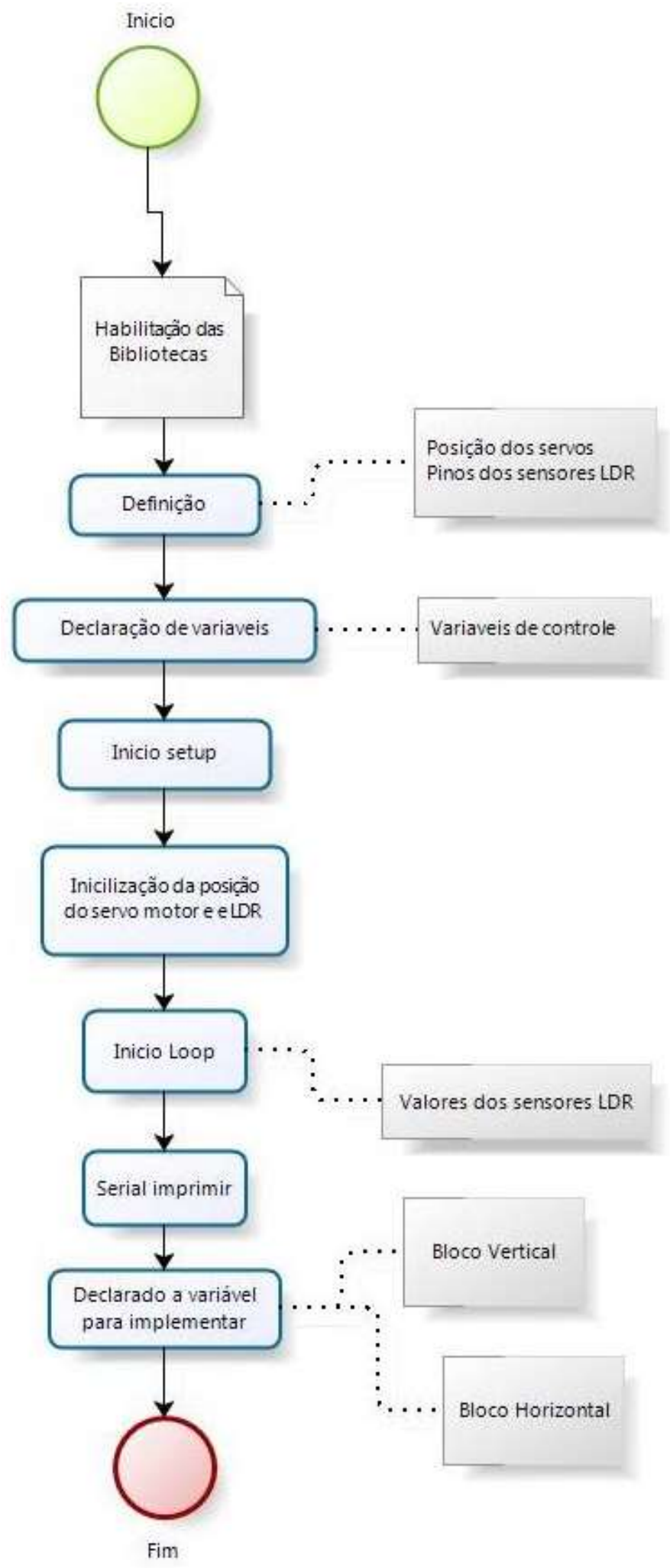

Figura 13: Fluxograma do código de sistemas de rastreamento solar. (Elaborada pelo autor, 2016).

\subsection{Bancada didática para ensaios de PV}

O protótipo intitulado de bancada didática foi construído e instalado para realização de experimentos e testes, tais como: 
- Medição da tensão e da corrente dos circuitos durante um período com aquisição a cada $10^{\circ}$;

- Medição de módulos fotovoltaico com a tensão 5v, 300mA e 1,5w com acoplamento em dois módulos em paralelo;

- Medição da energia e da potência fornecidas pelo protótipo de sistema rastreamento solar utilizando a interface no Software Excel;

- Medição da radiação e da potência fornecida.

\subsection{Teste com rastreamento móvel no laboratório.}

Na realização desse teste é utilizada uma bancada didática. O protótipo é colocado na bancada com o painel posicionado paralelamente ao plano da bancada (posição inicial).

Após isto, é ligada a alimentação do rastreamento, que se dá por uma fonte ajustável. Em seguida, a haste mecânica é deslocada no sentido anti-horário até o ângulo onde se deseja iniciar os ensaios (no caso, $5^{\circ}$ ). Então, depois de apagada a luz da sala, é ligada a luz de halogêneo da bancada didática.

Da posição inicial o protótipo rastreia a luz através dos sensores e desloca-se até a incidência luminosa, ficando a placa com o painel fotovoltaico o máximo possível paralelo à fonte de iluminação. Esse paralelismo geralmente apresenta um erro, que se explica devido à sensibilidade do sensor.

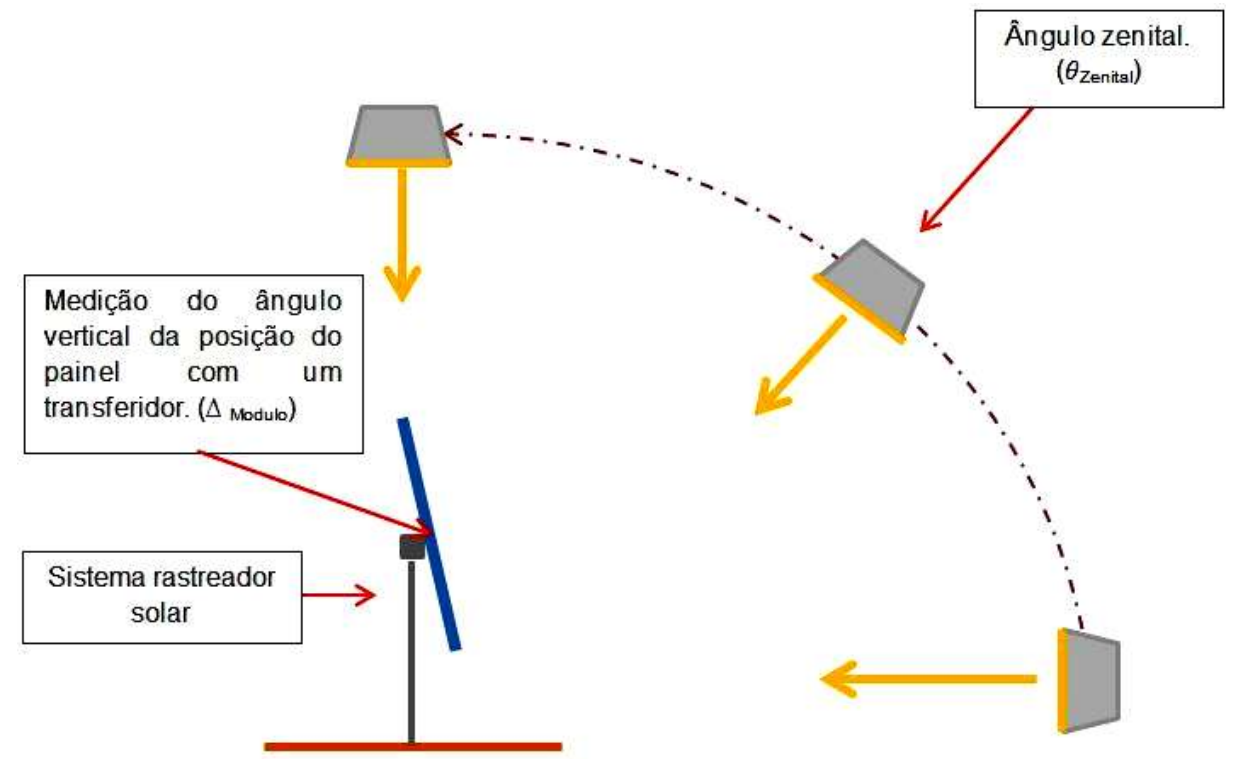

Figura 14: Geometria do teste com rastreamento na bancada didática.

(Elaborada pelo autor, 2016).

O erro de paralelismo é dado pela equação 4.

$$
\Delta_{\text {módulo }}=\left|\frac{\text { Angulo zinital }}{\text { Inclinação do pv }}\right|
$$


O valor dessa equação é sempre positivo, uma vez que o que se busca é a variação e ela é sempre positiva, daí a equação 4 vir na forma de módulo.

\subsection{Medição}

As medições de captação máxima e mínima foram feitas no PV para que se pudesse avaliar a influência da inclinação na captação de energia. O esquema pode ser conferido na Figura 14, verifica-se a tensão de circuito do painel solar para simulação do sistema fotovoltaico que pode ser medida em diversas variáveis: corrente $(I c c)$, tensão $(V c c)$, potência $(W)$, para medição de energia solar depende do valor de insolação em paralelo com diodo e em paralelo com um resistor R1 e painéis solares em série. A Figura 15 representa o diagrama de uma bancada com equipamento de medição. Uma vez definida a intensidade da luz é alterada a insolação. O melhor posicionamento da placa será a solução do problema, ou seja, a captação máxima de energia (Yilmaz, 2015).

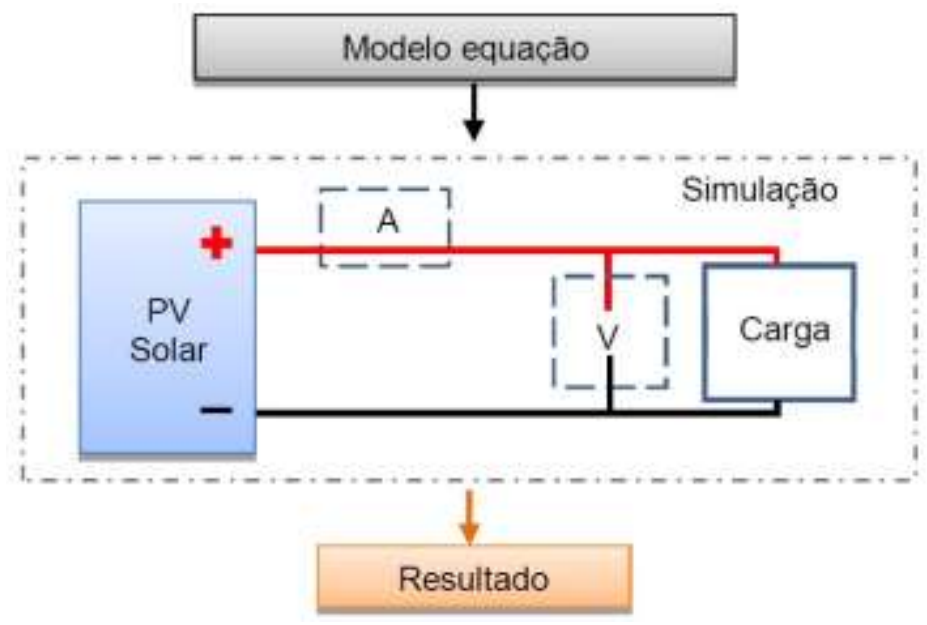

Figura 15: Diagrama de bloco do circuito elétrico para medição a painel fotovoltaico.

(Elaborada pelo autor, 2016).

O equipamento de medição está conectado em paralelo com o voltímetro como foi descrito anteriormente (Figura 15). Ao finalizar as primeiras medições, a chave é aberta e os reostatos ficam em série para as próximas medições com correntes menores. Segue a Equação 5:

$\mathrm{P}_{\mathrm{W}}=\mathrm{V}_{\mathrm{cc}} \cdot \mathrm{I}_{\mathrm{cc}}$

Em que,

Icc - corrente de curto-circuito $(\mathrm{A})$;

$V c c$ - tensão em circuito aberto (V);

$P w$ - potência no ponto de máxima (w). 


\section{RESULTADOS E DISCUSSÕES}

\subsection{Resultados}

A seguir são mostrados os resultados da geração de potência elétrica obtida pelo sistema com o uso da placa de sombreamento.

Simulando o Sol, foi colocada uma luz de halogêneo 300w na bancada didática que proporciona vários efeitos de iluminação, devido a um variador de luminosidade presente na mesma.

Resultados da resposta do sistema de rastreamento:

Os testes foram realizados conforme especificado, utilizando o sistema fotovoltaico dotado com rastreamento. Uma variação da posição da haste (onde estava fixada a lâmpada) foi realizada através do movimento mecânico e manual, obtendo-se posições para o ângulo zenital $\left(\vartheta_{z}\right)$ da bancada.

A tabela 1 apresenta tanto o tempo de resposta à mudança de posição do sistema de rastreamento quanto o erro de paralelismo entre a posição do painel fotovoltaico (com controlador de posição) e a posição da lâmpada.

Tabela 1: Ensaio do sistema de rastreamento.

\begin{tabular}{|c|c|c|c|c|}
\hline \multicolumn{5}{|c|}{ Ensaio sentido Anti-Horário } \\
\hline $\begin{array}{c}\text { Ângulo de } \\
\text { Incidência }\left({ }^{\circ}\right)\end{array}$ & $\begin{array}{c}\text { Tempo de } \\
\text { Resposta (seg.) }\end{array}$ & $\begin{array}{l}\text { Inclinação } \\
\text { do } P V\left({ }^{\circ}\right)\end{array}$ & $\begin{array}{l}\text { Variação } \\
\text { de Luz/PV }\end{array}$ & $\begin{array}{c}\text { Erro } \\
\text { de paralelismo \% }\end{array}$ \\
\hline 5 & 37 & 16 & 11 & \\
\hline 15 & 8 & 16 & 1 & $7 \%$ \\
\hline 25 & 7 & 16 & 9 & $36 \%$ \\
\hline 35 & 3 & 23 & 12 & $34 \%$ \\
\hline 45 & 4 & 34 & 11 & $24 \%$ \\
\hline 55 & 8 & 43 & 12 & $22 \%$ \\
\hline 65 & 7 & 57 & 8 & $12 \%$ \\
\hline 75 & 6 & 64 & 11 & $15 \%$ \\
\hline 85 & 6 & 68 & 17 & $20 \%$ \\
\hline 90 & 9 & 75 & 15 & $17 \%$ \\
\hline 85 & 7 & 75 & 10 & $12 \%$ \\
\hline 75 & 12 & 70 & 5 & $7 \%$ \\
\hline 65 & 6 & 65 & 0 & $0 \%$ \\
\hline 55 & 5 & 40 & 15 & $27 \%$ \\
\hline 45 & 4 & 30 & 15 & $33 \%$ \\
\hline 35 & 4 & 20 & 15 & $43 \%$ \\
\hline 25 & 10 & 17 & 8 & $32 \%$ \\
\hline 15 & 3 & 16 & 1 & $7 \%$ \\
\hline 5 & 3 & 15 & 10 & \\
\hline
\end{tabular}




\subsection{Discussão}

A linha de cor vermelha representa o maior erro de paralelismo entre o ângulo zenital e o PV. Este valor apareceu com maior frequência para o ângulo de (-) 35․ O menor erro de paralelismo entre os ângulos zenital e PV apareceu com maior frequência para o ângulo de 65으, representado pela cor azul, com um valor de $0 \%$.

Para o ângulo de incidência de 90 , observa-se que nos ensaios realizados, o maior valor para o erro de paralelismo foi de $17 \%$ e o menor, $12 \%$.

O tempo de resposta médio, para os seis ensaios, foi de 30,5 segundos. O tempo de resposta indica o deslocamento do painel solar com rastreamento (na posição de $90^{\circ}$ ) até a posição inicial dos testes que era de 5 o (ângulo de incidência).

\subsection{Performance elétrica}

Para obtenção dos resultados da performance elétrica foram realizados três ensaios. Em cada ensaio o ângulo zenital percorria o intervalo de $5^{\circ}$ até o ângulo de $175^{\circ}$, isso variando de $10^{\circ}$ em $10^{\circ}$, isto para o sistema com rastreamento solar.

\subsubsection{Tensão e Corrente}

A seguir são apresentados os gráficos da tensão $(V c c)$ e corrente $(I c c)$ em função do ângulo zenital do rastreamento.

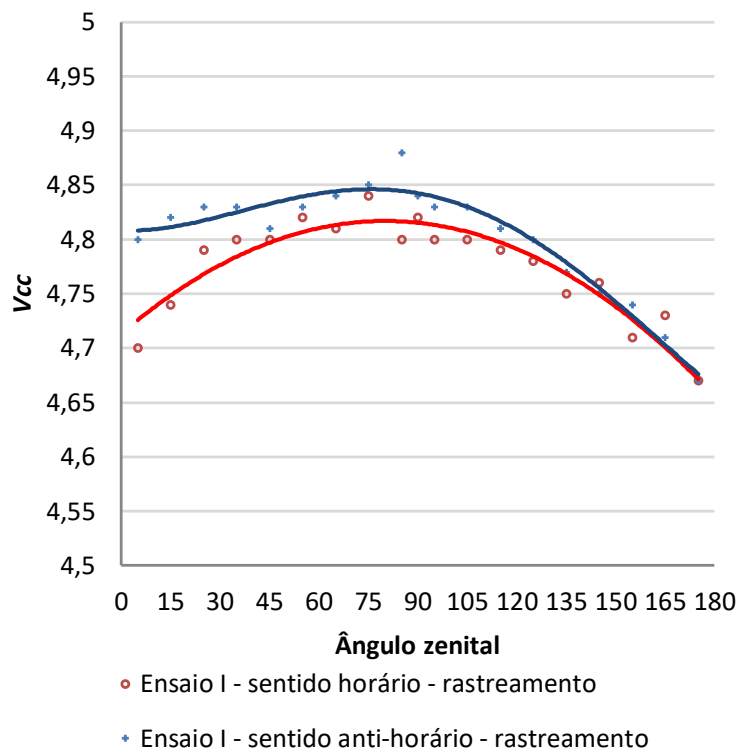

a)

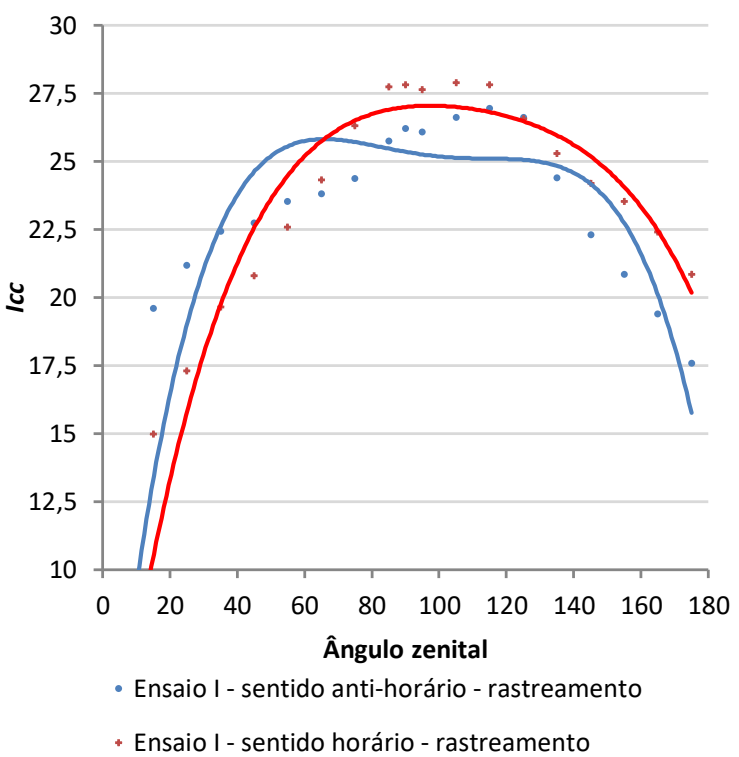

b)

Figura 16: Ensaio de histerese entre os resultados da inclinação no sentido anti-horário (azul) e horário (vermelho) é pequena, sendo: a) Tensão x Ângulo zenital; b) Corrente x Ângulo zenital.

\subsubsection{Potência gerada}

Para medição da potência gerada foi multiplicado o valor de Icc com o valor de Vcc. O resultado da variação da potência em função do ângulo zenital é mostrado no gráfico a seguir na Figura 17. 


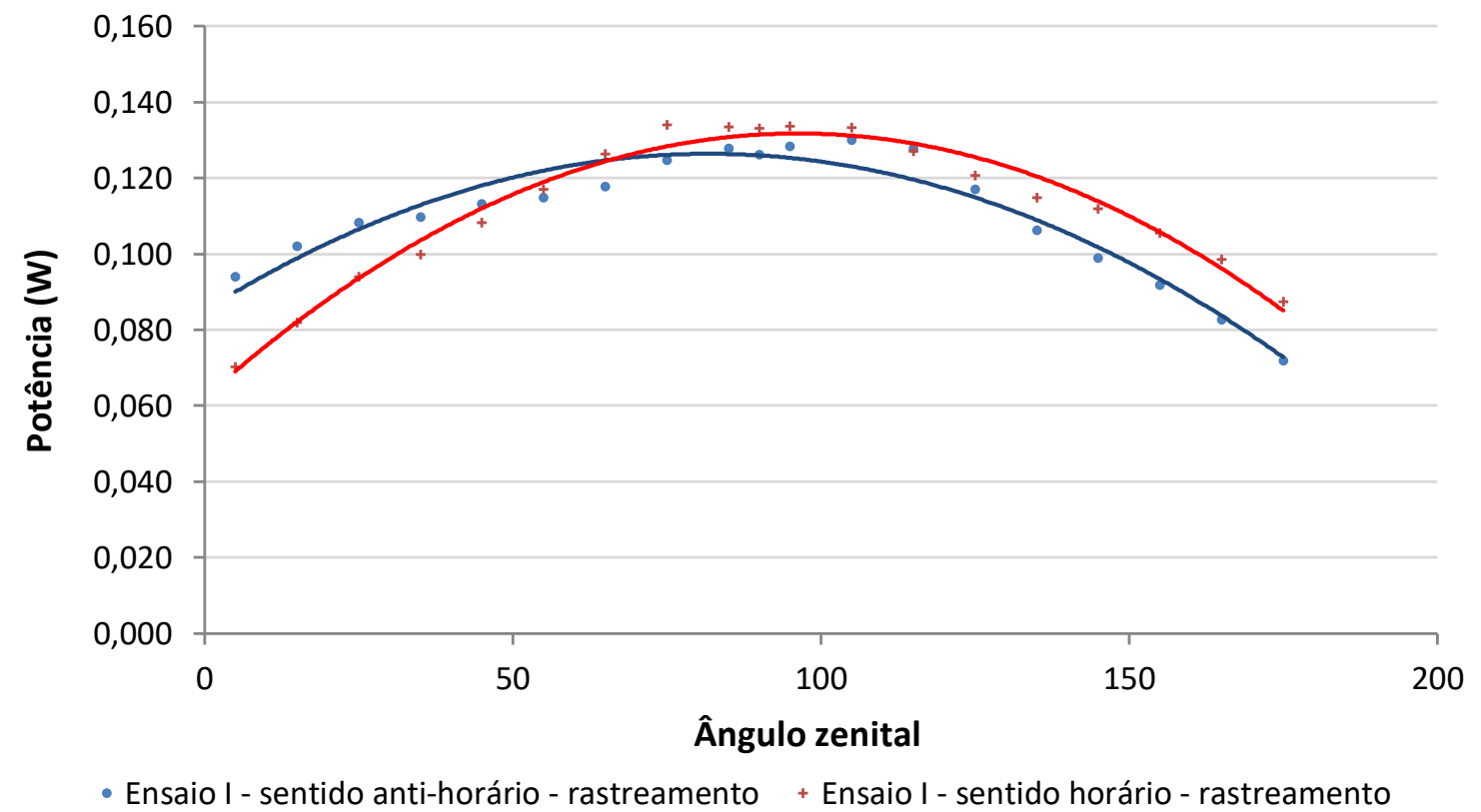

Figura: 17: Potência x Incidência.

\section{CONCLUSÃO}

A pesquisa realizada atingiu os objetivos propostos, dentre os quais a implantação de um protótipo, o projeto e a implementação de protótipo: placa de sombreamento em "LDR" para painel fotovoltaico utilizando microcontrolador.

Se considerarmos o maior valor de potência produzida, o sistema rastreável produz, aproximadamente, 2,5 (duas vezes e meia) mais energia que o fixo. Vale destacar que esse é o valor de potência máxima e não o valor de potência efetiva junte-se a isto o fato que a energia elétrica, necessária para alimentar o rastreamento automático, não foi diminuída.

A tecnologia simplificada, o baixo custo, além da avaliação do ganho na conversão da energia solar em energia elétrica que este sistema com controlador rastreável apresenta em relação a um sistema fixo é como esperado, seguindo os resultados encontrados na bibliografia.

\section{AGRADECIMENTOS}

Os autores agradecem à CAPES, Universidade Federal de Rio Grande do Norte - UFRN e ao Programa de Pós-Graduação em Engenharia Mecânica - PPgEM/Centro de Tecnologia da UFRN pelo suporte financeiro da pesquisa.

\section{REFERÊNCIAS}

Alcântara, S. M. F. (2010). Desempenho de um Painel Fotovoltaico de Baixa Tensão, Acoplado ao Protótipo de um Conversor DC/DC Topologia Booste. Natal - RN, Universidade Federal do Rio Grande do Norte - UFRN. Disponível em: 
<http://repositorio.ufrn.br:8080/jspui/bitstream/123456789/15632/1/saramfa.pdf>. Acesso em: 5 Jun. 2016.

Alves, A. F. (2008). Desenvolvimento de um Sistema de Posicionamento Automático para Painéis Fotovoltaicos. Botucatu - São Paulo. Universidade Estadual Paulista - UNESP. Disponível em: <http://www.pg.fca.unesp.br/teses/pdfs/arq0343.pdf>. Acesso em: 28 Abr. 2016.

Cortez, R. J. M. (2013). Sistema de Seguimento Solar em Produção de Energia Fotovoltaica. Porto Portugal. Universidade do Porto. Disponível em: <http://paginas.fe.up.pt/ ee06279/docs/dissertacao.pdf> Acesso em: 27 Maio 2016.

EPE. (2012). Análise da Inserção da Geração Solar na Matriz Elétrica Brasileira. Rio de Janeiro: Empresa de Pesquisa Energética.

M. G. Villalva, J. R. Gazoli. Energia Solar Fotovoltaica - Conceitos e Aplicações - Sistemas Isolados e Conectados à Rede. 1‥ Ed. ano 2012. São Paulo: [s.n.].

Medeiros, I. P. M, Fontes, F. A. O, Lima, C. M, Barbosa, C. R. F, Valcacer, S. M, Sanabio R. G, Dantas, V. B. (2015). Uso de Sistemas Automatizados para Otimizar a Captação de Energia em Painéis Solares. Congresso nacional de matemática aplicada à indústria, v. 1, n. 1, p. 8. dx.doi.org/10.5151/mathpro-cnmai-0006.

Miyashiro, R. F.; Ottoboni, R. (2014). Sistema de Posicionamento para Coletores Solares Residenciais de Materiais Recicláveis. Revista científica da FEPI, p. 4. ISSN: 2175-4020.

Moraes, E. P. (2012). Seguidor Solar de um Único Eixo Inclinado. Brasília - DF. Centro Universitário de Brasília - UNICEUB. Disponível em: <http://www.repositorio.uniceub.br/bitstream/235/3636/3/monografiaeulermoraes-22012.pdf>. Acesso em: 1 Jun. 2016.

Paiva, E. C. (2009). Desenvolvimento de um Rastreador Solar Microcontrolador para um Coletor Solar Concentrador. Viçosa - MG. Universidade Federal de Viçosa - UFV. Disponível em: <http://www.locus.ufv.br/handle/123456789/737>. Acesso em: 5 Maio. 2016.

Rodrigues, P. R. (2014). Projeto de Bancada Didática Experimental para o Uso de Energia Solar e Eólica. Brasília - DF. Universidade de Brasília - UnB. Disponível em: <http://bdm.unb.br/bitstream/10483/9263/1/2014_pedrorabelorodrigues.pdf>. Acesso em: 8 Maio. 2016.

Silva, A. L. M; Cutin, M. S.; Machado, S. G. (2001). Sistema de Levitação Eletromagnético Controlado por Computador. Rio de Janeiro, RJ. Universidade do Estado do Rio de Janeiro - UERJ. Disponível em: <http://www.lee.eng.uerj.br/ jpaulo/pg/2001/pg-levitacao-2001.pdf> Acesso em: 27 Maio 2016.

Silva, V. R. (2014). Uma Proposta para Automatização do Monitoramento da Interceptação Luminosa em Pastagens Utilizando uma Rede de Sensores Sem Fio de Baixo Custo. Goiânia GO. Universidade Federal de Goiás - UFG. Disponível em: <https://repositorio.bc.ufg.br/tede/handle/tede/3549>. Acesso em: 22 Maio 2016. 
Tobergte, D. R.; Curtis, S. (2013). Atlas de Energia Elétrica do Brasil. Brasília - DF: ANEEL. V. 53.

Trevelin, F. C. (2014). Estudo Comparativo Entre Métodos de Rastreamento Solar Aplicado a Sistemas Fotovoltaicos. São Paulo. Universidade de São Paulo - USP. Disponível em: <http://www.tcc.sc.usp.br/tce/disponiveis/18/180450/tce-02092014-093245/?\&lang=br>. Acesso em: 2 Jun. 2016.

Yilmaz, S.; Ozcalik, H. R.; Dogmus, O.; Dincer, F.; Akgol, O.; Karaaslan, M. (2015). Design of Two Axes Sun Tracking Controller With Analytically Solar Radiation Calculations, Renewable and Sustainable Energy Reviews, Volume 43, March, p. 997-1005, ISSN 1364-0321, http://dx.doi.org/10.1016/j.rser.2014.11.090. 